\title{
A sustained, non-insulin related, hypoglycaemic effect of electroacupuncture in diabetic Psammomys obesus
}

\author{
M. Y. Shapira ${ }^{1}$, E. Y. Appelbaum ${ }^{2}$, B.Hirshberg ${ }^{1}$, Y.Mizrahi ${ }^{2}$, H. Bar-On ${ }^{2}$, E.Ziv $^{2}$ \\ ${ }^{1}$ Division of Internal Medicine, Hadassah University Hospital, Hebrew University - Hadassah Medical School, Jerusalem, Israel \\ ${ }^{2}$ Diabetes Research Unit, Hadassah University Hospital, Hebrew University - Hadassah Medical School, Jerusalem, Israel
}

\section{Abstract}

Aim/hypothesis. Electroacupuncture has been shown to induce a short-term hypoglycaemic effect in streptozotocin diabetic rats. We designed an experiment to investigate the effect of electroacupuncture in Psammomys obesus, a model of insulin resistance and non-insulin-dependent diabetes mellitus.

Methods. We divided 29 diabetic Psammomys randomly into three groups: abdominal electroacupuncture (real, $n=11$ ), back electroacupuncture (placebo, $n=9$ ) and control (anaesthesia, $n=9$ ). Electroacupuncture was carried out on days 1,3 and 5 of the experiment. During the first week of the experiment, blood glucose was tested three times on treatment days and once on the following days. Over the next 2 weeks, blood glucose was tested every other day. Animals were weighed at the same time of blood sampling. After 3 weeks, at the end of the experiment, blood was drawn for measurement of insulin, fructosamine, cholesterol and triglycerides.

Results. At day 5 (end of intervention), blood glucose (as per cent of primary concentrations, means \pm SE) was $57 \pm 10,93 \pm 13$ and $89 \pm 11$ for the real, placebo and control groups respectively $(p=0.02)$. At day 8 , blood glucose $68 \pm 14,86 \pm 16$ and $97 \pm 9$ for the real, placebo and control groups respectively $(p=0.04)$. At day 22, blood glucose was $79 \pm 11,85 \pm 15$ and $131 \pm 2$ for the real, placebo and control groups $(p=0.04)$. Comparison of the decline in blood glucose, throughout the 3 weeks, between the real and placebo groups by ANOVA was highly significant $(p<0.0001)$, the difference between the placebo and control groups at the same time was not significant $(p>0.05)$. Animal weight gain, serum insulin, fructosamine, cholesterol and triglycerides were not significantly different between real and placebo groups. Conclusion/interpretation. Electroacupuncture at special abdominal acupoints induces a sustained hypoglycaemic effect in diabetic Psammomys compared with electroacupuncture at non-specific points, without weight loss. No hypoinsulinaemic effect was shown in the real and placebo groups. [Diabetologia (2000) 43: 809-813]

Keywords Chinese medicine, acupuncture, alternative medicine, diabetes mellitus, Psammomys obesus, insulin resistance.
Received: 15 November 1999 and in revised form: 24 January 2000

Corresponding author: M. Shapira MD, Bone Marrow Transplantation Unit, Hadassah University Hospital, PO Box 12000, Jerusalem, Israel, 91120

Abbreviations: TCM, Traditional Chinese medicine; EA, electroacupuncture; REA, real EA; PEA, placebo EA; C, control; STZ, streptozotocin.
Diabetes mellitus is a common disease that carries a high rate of late complications, often leading to death [1]. New innovative approaches of treatment are sought after intensively. Traditional Chinese medicine (TCM) claims to influence diabetes by acupuncture and herbal medicine. Belief and centuries of personal experience have supported these claims. The American Diabetes Association warns about the use of unproven therapies in diabetes care and called for basic and clinical research using well-established principles of investigation [2]. 
Table 1. Animal characteristics at the beginning of the experiment (means \pm SEM)

\begin{tabular}{lccc}
\hline & REA & PEA & C \\
\hline$n$ & 11 & 9 & 9 \\
Age (days) & $167 \pm 3$ & $168 \pm 4$ & $153 \pm 15$ \\
Weight (gram) & $241 \pm 14$ & $235 \pm 9$ & $236 \pm 16$ \\
Mean basal glucose & & & \\
concentrations (mg/dl) & $18.1 \pm 1.7$ & $17.9 \pm 1.3$ & $18.1 \pm 1.2$ \\
\hline
\end{tabular}

$\overline{\mathrm{REA} \text { - real electroacupuncture, PEA - placebo electroacu- }}$ puncture, $\mathrm{C}$ - control

Recently, a study was published [3] about a shortacting hypoglycaemic effect of electroacupuncture (EA) in streptozotocin (STZ)-induced diabetes in rats.

Psammomys obesus ("sand rat") is a desert gerbil lapsing into hyperinsulinaemia and hyperglycaemia when eating a laboratory rodent diet instead of its native food [4]. Genetic factors predispose it to insulin resistance and Type II (non-insulin-dependent) diabetes mellitus [5], whereas environmental, mainly nutritional, factors contribute to the expression of the diabetic phenomena. Primary insulin resistance in the Psammomys obesus leads to the development of hyperglycaemia [6]. The progression into nutritionally induced diabetes resembles in many respects the development of insulin resistance and Type II diabetes in certain human populations.

The Psammomys is a well-defined model and recently the colony was divided into two genetic lines: diabetes prone (DP) and diabetes resistant (DR) lines [7]. The transition of Psammomys of the DP line, to diabetes, when fed a high energy (HE) diet has been classified into four, often consecutive states [8]: A: normoinsulinaemic and normoglycaemic; B: hyperinsulinaemic and normoglycaemic; C: hyperinsulinaemic and hyperglycaemic and D: hyperglycaemic and hypoinsulinaemic. Hyperglycaemia and hypoinsulinaemia in stage $\mathrm{D}$ is due to the loss of betacell insulin secretion. It is possible to show a sustained hypoglycaemic effect of potential drugs in the Psammomys model, for example the hypoglycaemic effect of vanadyl sulphate in this model [9].

In this study we evaluated the immediate and prolonged hypoglycaemic effect of EA in diabetic Psammomys obesus.

\section{Materials and methods}

Animals. We used 29 diabetic male Psammomys obesus of the diabetes prone (DP) line, from Harlan, Jerusalem, Israel. Diabetes was induced by a high energy (HE) diet (Kofolok, Petach Tikva Israel, $3.16 \mathrm{kcal} / \mathrm{g}$ ). Blood glucose above $11.1 \mathrm{mmol} / \mathrm{l}$ was verified by two separate blood samples before entering the experiment. The animals were randomly assigned into three groups: abdominal EA (real-REA), back EA (placebo-PEA) and control (anaesthesia only-C). Characteristics of the animals in each group are listed in Table 1 . All animals were anaesthetised with i.p. penthotal $6 \%(60 \mathrm{mg} / \mathrm{kg})$ during EA for $1 \mathrm{~h}$. Control animals were anaesthetised at the same time intervals as the above groups. All experimental procedures were authorised by the institutional animal care committee.

Electroacupuncture. We treated REA animals at CV12 (Zhongwan - located at the middle point between the lower edge of the xyphoid and the umbilicus) and CV4 (Gwanyuan - located at midline, 3 cun [a cun is a Chinese term for relative body measure that is specifically determined in every patient] below the umbilicus) acupuncture points [10] with sterile acupuncture needles (Suzhou medical instruments factory, Suzhou, China). The placebo group (PEA) was treated at parallel points to the abdominal points in the para-vertebral region. Acupuncture needles were inserted approximately $2 \mathrm{~mm}$ into the muscular layer. We carried out EA on days 1, 3 and 5 of the experiment for $30 \mathrm{~min}$ per treatment at a current of $80 \mathrm{mAmp}$ (which was chosen according to our experience in the Psammomys [M.Y. Shapira et al., unpublished results]) and frequency of $15 \mathrm{~Hz}$ (Bio-tech AWQ-104, China).

Experimental protocol. During the first week of the experiment, blood glucose was tested three times on the treatment days to evaluate the immediate response to EA (before anaesthesia, after anaesthesia but before treatment and immediately after treatment) and the following days. During the next 2 weeks, blood glucose was tested every other day. Animals were weighed at the same time of blood sampling. After 3 weeks at the end of the experiment, blood was drawn for measurement of insulin, fructosamine, cholesterol and triglycerides.

Assays. We measured blood glucose on 3 microlitre samples with Glucometer Elite (Bayer, Elkhart, Ind., USA). Plasma triglycerides, cholesterol and fructosamine were measured by enzymatic methods (Roche, Basel, Switzerland). Plasma insulin was measured by radioimmunoassay, utilising anti-human insulin antibodies (Sorin Biomedica, Saluggia, Italy) and human insulin as standards.

Statistical analysis. All concentrations are given as means \pm SEM. Group comparisons were made by paired Student's $t$ test and analysis of variance (ANOVA). A significant difference was considered $p<0.05$.

\section{Results}

Long-term effect. Blood glucose concentrations during the 3 weeks of experiment are shown in Fig. 1. To eliminate a variability in glucose concentrations, comparison of blood glucose is presented as a per cent of basal concentrations. Mean glucose concentrations at the beginning of the experiment were $18.1 \pm 1.7,17.9 \pm 1.3$ and $18.1 \pm 1.2 \mathrm{mmol} / \mathrm{l}$ for REA, PEA and C respectively. Note the stepwise cumulative hypoglycaemic effect during days $1-6$, and the sustained effect until the end of the experiment. Comparison of the control group with the RE group is consistently significant ( $p$ between 0.02 and 0.05 ). 


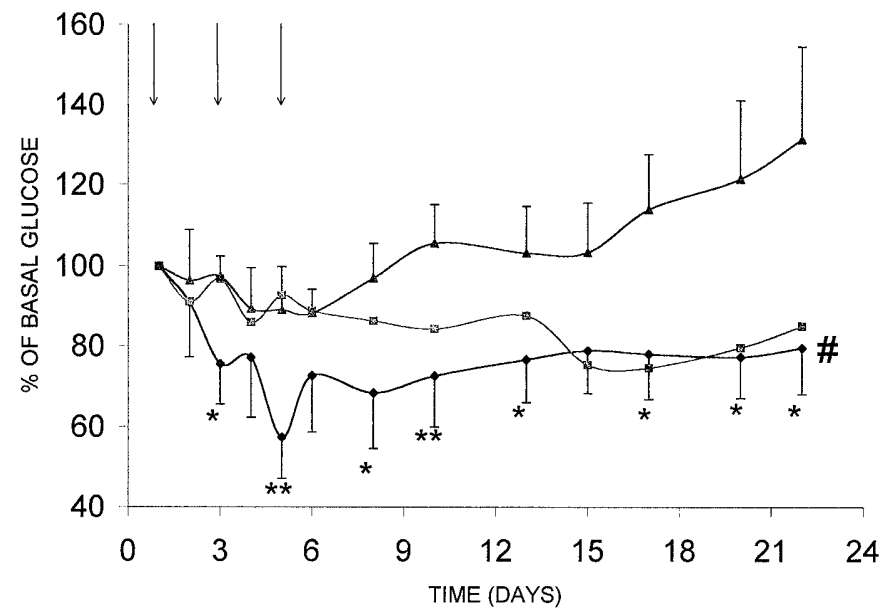

Fig. 1. Comparison of mean blood glucose (expressed as per cent of basal concentrations) in diabetic Psammomys obesus following real electroacupuncture (REA), placebo electroacupuncture (PEA) or control (C). Arrows indicate therapeutic session. $\# p<0.001 ; * p<0.05 ; * * p<0.02 ; \multimap, \mathrm{REA} ;-\square$, $\mathrm{PEA} ; \boldsymbol{\Lambda}, \mathrm{C}$

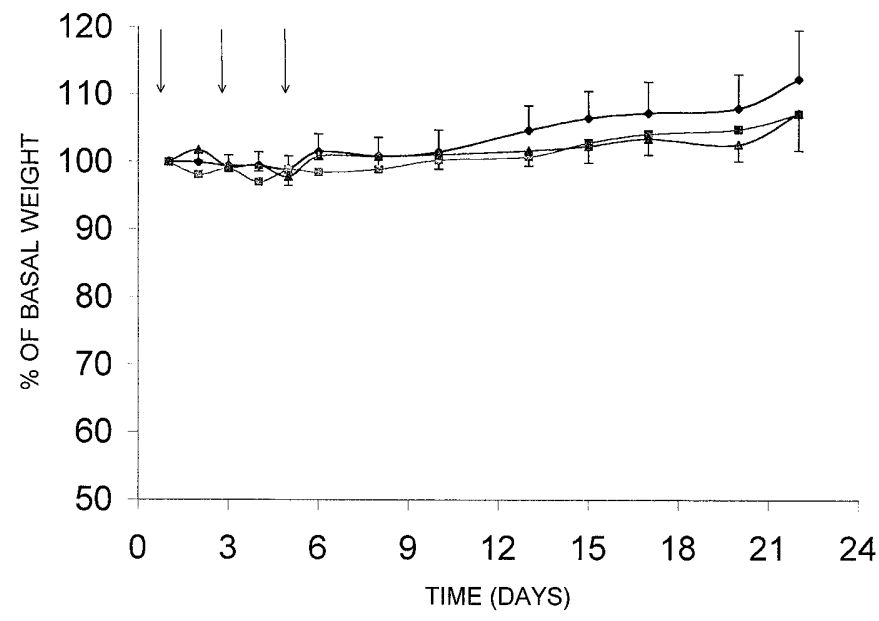

Fig. 2. Comparison of weight gain during the study period in the real electroacupuncture (REA), placebo electroacupuncture (PEA) or control (C) Psammomys. - , REA; $-\mathbf{-}$, $\mathrm{PEA} ; \boldsymbol{\Lambda}, \mathrm{C}$

Comparison of REA and PEA by ANOVA was highly significant $(p<0.0001)$, the difference between PEA and $\mathrm{C}$ at the same time was not significant $(p>0.05)$. Animal weight gain is shown in Figure 2. Animals in all groups gained weight and there was no significance between groups.

Immediate effect. Blood glucose concentrations as measured before and immediately after EA treatment are shown in Table 2. No significant immediate hypoglycaemic effect of EA was noted. Average serum insulin, fructosamine, cholesterol and triglycerides of the surviving animals in each group $(9,6$ and 6 animals in the REA, PEA and C groups respective-
Table 2. Short-term effect ( $30 \mathrm{~min}$ after treatment) of electroacupuncture. [Blood glucose concentrations in $\mathrm{mmol} / \mathrm{l}$ (means \pm SEM)]

\begin{tabular}{llcc}
\hline Group & $\begin{array}{l}\text { Glucose before } \\
\text { anaesthesia }\end{array}$ & $\begin{array}{l}\text { Glucose after } \\
\text { EA treatment }\end{array}$ & Change (\%) \\
\hline REA & $18.1 \pm 1.7$ & $17 \pm 2.4$ & $-4 \pm 12$ \\
PEA & $17.9 \pm 1.3$ & $16.3 \pm 2.2$ & $-4 \pm 11$ \\
C & $18.1 \pm 1.2$ & $21.2 \pm 2.7$ & $+19 \pm 16$ \\
\hline
\end{tabular}

REA - real electroacupuncture, PEA - placebo electroacupuncture, $\mathrm{C}$ - control.

All results were statistically non-significant

Table 3. Average serum insulin, fructosamine, cholesterol and triglycerides of the surviving animals in each group at the end of the experiment (means \pm SEM)

\begin{tabular}{lccc}
\hline & REA & PEA & C \\
\hline Insulin $(\mathrm{pmol} / \mathrm{l})$ & $2460 \pm 630$ & $2706 \pm 1374$ & $1296 \pm 462$ \\
Fructosamine $(\mathrm{mmol} / \mathrm{l})$ & $2.13 \pm 0.16^{\mathrm{a}}$ & $2.88 \pm 0.83$ & $2.65 \pm 0.31$ \\
Cholesterol $(\mathrm{mmol} / \mathrm{l})$ & $3.7 \pm 1$ & $4.2 \pm 2.1$ & $2.6 \pm 0.4$ \\
Triglycerides $(\mathrm{mmol} / \mathrm{l})$ & $3.2 \pm 0.8$ & $3.9 \pm 0.9$ & $3.3 \pm 0.5$ \\
\hline
\end{tabular}

REA - real electroacupuncture, PEA - placebo electroacupuncture, $\mathrm{C}$ - control.

Number of surviving animals $-9,6$ and 6 in the REA, PEA and $\mathrm{C}$ groups respectively.

${ }^{\mathrm{a}} p=0.058$ when compared to $\mathrm{C}$

ly) at the end of the experiment ( 3 weeks after initiation of EA and 17 days after the last treatment) are shown in Table 3. There was hyperinsulinaemia in the REA and PEA groups without a significant difference. A trend toward lower fructosamine in REA animals was noted $(p=0.058)$ when compared with $\mathrm{C}$ animals.

\section{Discussion}

Diabetes mellitus is a chronic disorder of an impaired metabolism of glucose caused by partial or complete deficiency of insulin or insulin resistance or both, and includes development of vascular and neuropathic complications. Primary complications result from insulin deficiency and later complications result from consistent hyperglycaemia. Diabetes is one of the commonest causes of morbidity and mortality in the western world [1]. Conventional treatment for Type II diabetes is based on the combination of attenuated diet, weight loss, exercise and drugs. A need for extension of therapeutic measures is stressed, especially for treatments including long-acting and reduced insulin consumption effect.

Traditional Chinese medicine is based on an assumption that a balance between two forces (yin and yang) is the key to good health. An imbalance of either force might cause illness. Balance can be restored by various methods including exercise, mas- 
sage, acupuncture and herbs. Belief and centuries of personal experience support this theory. Claims that diabetes can be influenced by acupuncture and herbal medicine exist in Chinese literature $[11,12]$ but few scientific records on the efficacy of these methods exists $[13,14]$. Electroacupuncture is a technique designed to amplify the effect achieved by ordinary acupuncture. In this technique, an electric power source is connected to the needles after their insertion. The current and frequency used depend on the nature of the disease treated.

In this study we investigated the effect of EA on a model of Type II diabetes, Psammomys obesus, which mimics the nutritionally induced, human population-based diabetes. We showed a prolonged hypoglycaemic effect of EA in CV12 and CV4 in this model of nutritionally induced insulin resistance and Type II diabetes that remained sustained for more than 2 weeks after the end of the therapeutic intervention (Fig.1). This effect was significantly different from the effect of EA in non-specific points (PEA group) or if only anaesthesia was given (control group). The difference between REA and PEA groups indicates that the hypoglycaemic effect is not only related to electric stimulation of muscular or adipose tissue, but is also dependent on location. Thus, the assumption that non-specific stimulation of these tissues induces a hypoglycaemic effect [3] does not correspond with our results. In the Psammomys, a reduction in appetite induces a decrease in body weight and glucose concentrations. Our results indicate that the hypoglycaemic effect was not related to appetite since the animals' weight increased during the experimental period (Fig.2). Another study [3] has recently shown a short-acting (30 min) hypoglycaemic effect of EA in a model of STZ-induced diabetes mellitus in rats. The hypoglycaemic effect was related to increased endorphins and was blocked by naloxon. It was hypothesised that the hypoglycaemic action of EA was related to stimulation of insulin secretion, since it disappeared in rats with insulin resistance induced by repeated injections of long-acting insulin. This hypothesis was also supported by an increase in plasma insulin-like immunoreactivity after EA stimulation in normal rats. We showed a sustained effect which cannot be related to neural endorphin release since there was no immediate change in glucose concentrations after EA and the hypoglycaemic effect was present over 2 weeks after the termination of the therapeutic intervention. Hyperinsulinaemia was present in the REA and PEA groups without a significant difference, which establishes a non-direct insulin effect. Several of the animals in the control group entered stage D (hyperglycaemic and hypoinsulinaemic) of diabetes [8], thus explaining the increase in plasma glucose and decrease in insulin concentrations. The REA group showed a trend of returning from stage $\mathrm{C}$ (hyperinsulinaemic and hyperglycae- mic) to stage B (hyperinsulinaemic and normoglycaemic). Vanadyl sulphate was shown to induce a hypoglycaemic effect through more potent insulin action [9]. A therapeutic intervention of a few days with vanadyl induces the hypoglycaemic effect for a few weeks, which resembles the effect of EA. The hypoglycaemic effect in our system can be attributed to increased insulin sensitivity. This might be caused either by a decrease in hepatic gluconeogenesis similar to that occurring in older Psammomys [15] or by an increase in glucose uptake by the muscle tissue resembling Psammomys reaction to vanadyl sulphate [9] or a combination of both.

Hypertriglyceridaemia was found in all groups without a difference because of the hyperinsulinaemic state even with EA treatment. Fructosamine concentrations were not significantly different but more animals in the control and PEA group died of hyperglycaemia thus creating a bias since the fructosamine was measured at the end of the experiment only in the surviving animals.

We conclude that EA at specific acupoints (CV12 [Zhongwan] and CV4 [Gwanyuan]) in Psammomys obesus has a prolonged and sustained hypoglycaemic effect that is not related to food intake but to an increased insulin sensitivity.

Acknowledgements. We wish to thank Prof E. Shafrir for his advice during the research work and for his helpful discussion.

\section{References}

1. Sherwin RS (1995) Diabetes mellitus. In: Bennet CJ, Pulm F (eds), Cecil Textbook of Medicine, (20th edn.) WB Saunders, Philadelphia, pp 1258

2. ADA Clinical Practice Recommendations (1999) Position Statement - Unproven Therapies. Diabetes Care 22: S104

3. Chang SL, Lin JG, Chi TC et al. (1999) An insulin-dependent hypoglycaemia induced by electroacupuncture at the Zhongwan (CV12) acupoint in diabetic rats. Diabetologia 42: 250-255

4. Ziv E, Shafrir E (1995) Psammomys obesus: nutritionally induced NIDDM-like syndrome on the "thrifty gene" background. In: Shafrir E (ed) Lessons from Animal Diabetes, 5. Smith-Gordon, London, pp 285-300

5. Ziv E, Kalman R, Hershkop K, Barash V, Shafrir E, BarOn H (1996) Insulin resistance in the NIDDM model Psammomys obesus in the normoglycaemic, normoinsulinaemic state. Diabetologia 39: 1269-1275

6. Shafrir E, Ziv E (1998) Cellular mechanism of nutritionally induced insulin resistance: the desert rodent Psammomys obesus and other animals in which insulin resistance leads to detrimental outcome. J Basic Clin Physiol Pharmacol 9: 347-385

7. Kalman R, Adler JH, Lazarovici G, Bar-On H, Ziv E (1993) The efficiency of sand rat metabolism is responsible for development of obesity and diabetes. J Basic Clin Physiol Pharmacol 4: 57-68

8. Kalderon B, Gutman A, Levy E, Shafrir E, Adler JH (1986) Characterization of stages in development of obesi- 
ty-diabetes syndrome in sand rat (Psammomys obesus). Diabetes 35: 717-724

9. Ziv E, Kalman R, Bar-On H, Shafrir E (1996) Potentation of insulin action by vanadyl treatment in diabetic Psammomys obesus. Exp Clin Enocrinol Diabetes 104: S164-S165

10. The location of acupoints (1995) State Standard of the People's Republic of China. Delta Publishing Sdn Bhn, Selangor Darul Ehsan, Malaysia, pp 67-69

11. Feng M, Li Y, Pang B et al. (1997) Acupuncture combined with application of xiaoke plaster for treatment of 309 cases of diabetes mellitus. J Tradit Chin Med 17: 247-249

12. Hu H (1995) A review of treatment of diabetes by acupuncture during the past forty years. J Tradit Chin Med 15: $145-154$
13. Mo X, Chen D, Ji C et al. (1996) Effect of electro-acupuncture and transcutaneous electric nerve stimulation on experimental diabetes and its neuropathy. Chen Tzu Yen Chiu 21: 55-59

14. Chen D, Gong D, Zhai Y (1994) Clinical and experimental studies in treating diabetes mellitus by acupuncture. J Tradit Chin Med 14: 163-166

15. Ziv E, Shafrir E, Kalman R, Galer S, Bar-On H (1999) Changing pattern of prevalence of insulin resistance in Psammomys obesus, a model of nutritionally induced type 2 diabetes. Metabolism 48: 1549-1554 